\title{
Stability of Turing patterns in the Brusselator model
}

\author{
B. Peña and C. Pérez-García \\ Instituto de Física, Universidad de Navarra, E-31080 Pamplona, Spain \\ (Received 21 December 2000; revised manuscript received 18 June 2001; published 22 October 2001)
}

\begin{abstract}
The selection and competition of Turing patterns in the Brusselator model are reviewed. The stability of stripes and hexagons towards spatial perturbations is studied using the amplitude equation formalism. For hexagonal patterns these equations include both linear and nonpotential spatial terms enabling distorted solutions. The latter modify substantially the stability diagrams and select patterns with wave numbers quite different from the critical value. The analytical results from the amplitude formalism agree with direct simulations of the model. Moreover, we show that slightly squeezed hexagons are locally stable in a full range of distortion angles. The stability regions resulting from the phase equation are similar to those obtained numerically by other authors and to those observed in experiments.
\end{abstract}

DOI: 10.1103/PhysRevE.64.056213

PACS number(s): 05.45.-a, 82.40.Bj, 82.40.Ck

\section{INTRODUCTION}

Some chemical systems out of equilibrium undergo a spatial symmetry breaking, leading to stationary pattern formation on macroscopic scales [1]. The new stationary states form periodic concentration structures with a wavelength independent of the reactor geometry, the so-called Turing patterns [1]. Experimental evidence for Turing patterns was first obtained in 1990 by Castets et al. [2], in the well-known chlorite-iodine-malonic acid (CIMA) reaction, and later on in the chlorine-dioxide iodine-malonic acid (CDIMA) reaction [3,4], and in the polyacrilamide-methylene blue-oxygen reaction, althought in the latter the leading mechanism is still under discussion [5]. Depending on the control parameters (concentrations of reactants and diffusion coefficients), the dynamics of the CIMA or CDIMA reaction exhibits several kinds of steady patterns close to onset: stripes, hexagons, and "rhombs" [6]. (Usually, the so-called "black eyes" seem to arise from secondary bifurcations.) Realistic reactive schemes are, in general, quite complicated. Consequently they are replaced by simplified schemes, as the Brusselator model [7], reproducing the observed patterns while dealing with simple calculations [8-10].

In the present paper we will focus on stationary Turing patterns arising in the Brusselator. We discuss and obtain a generalized amplitude equation, including spatial modulations, for the planforms appearing in the model. This equation has been obtained in many chemical models for stripe patterns only, but the hexagonal case has not been discussed in detail so far [8-10]. From symmetry arguments, some authors established that it must include some nonpotential quadratic terms besides the usual diffusive linear one $[11,12]$. By means of a multiple-scale technique we compute the coefficients of the amplitude equation for the Brusselator. Stationary solutions of this equation are stripes, hexagons with two different phases, mixed modes, and distorted hexagons. A linear stability analysis sets the stability of these stationary solutions in regard to amplitude disturbances.

Another stability limit is obtained from phase perturbations. In fact, amplitude varies much more rapidly in time than phase does and, therefore, it becomes enslaved by the slowly varying phases. The slow dynamics of slight spatial heterogeneities in the pattern can be described with a phase equation for each stationary solution. The stability regions computed for the Brusselator can be considered as reminiscent of the Busse's balloon in thermal convection [13].

The paper is organized as follows. In Sec. II we introduce the two-dimensional (2D) model and we give a general survey of the linear analysis. In Sec. III we carry out a weakly nonlinear analysis using the multiple-scale method to derive the amplitude equations. The stability of patterns towards homogeneous perturbations (amplitude instabilities) is discussed in Sec. IV. The stability regions for roll, perfect hexagons, and squeezed hexagons are explicitly computed. We derive the linear phase equations for different kind of hexagons in Sec. V and we compare the stability diagrams obtained analytically with direct numerical simulations of the Brusselator model. Sec. VI summarizes our conclusions.

\section{THE REACTION-DIFFUSION MODEL}

The Brusselator is considered one of the simplest reaction-diffusion models exhibiting Turing and Hopf instabilities. The spatiotemporal evolution of the main variables is given by the following partial differential equations:

$$
\begin{gathered}
\partial_{t} X=A-(B+1) X+X^{2} Y+\nabla^{2} X, \\
\partial_{t} Y=B X-X^{2} Y+D \nabla^{2} Y,
\end{gathered}
$$

where $X$ and $Y$ denote the concentrations of activator and substrate, respectively. Here $D$ is a parameter proportional to the diffusion ratio of the two species $D_{Y} / D_{X}$ and, as usual, $B$ is kept as the control parameter of the problem.

The homogeneous steady state of these equations is simply $\mathbf{u}_{s}=\left(X_{s}, Y_{s}\right)=(A, B / A)$ [9]. Let us briefly recall here the results of the linear stability analysis around $\mathbf{u}_{s}$. Considering small perturbations $\mathbf{u}=(x, y)$ in Eqs. (1) one arrives at

$$
\partial_{t} \mathbf{u}=\mathcal{L} \mathbf{u}+\left(\frac{B}{A} x^{2}+2 A x y+x^{2} y\right)\left(\begin{array}{c}
1 \\
-1
\end{array}\right)
$$

where $\mathcal{L}$ is the linearized operator 


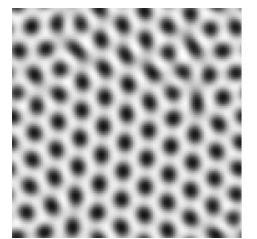

(a)

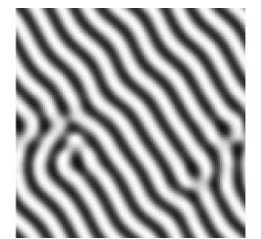

(b)

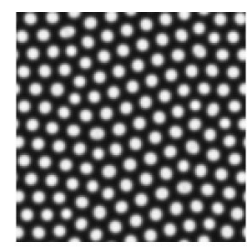

(c)
FIG. 1. Turing patterns from direct simulations of Brusselator model $(A=4.5, D=8)$ : (a) $\mu=0.04$ initial hexagons, (b) $\mu=0.30$ striped pattern, and (c) $\mu=0.98$ reentrant hexagons.

$$
\mathcal{L}=\left(\begin{array}{cc}
B-1+\nabla^{2} & A^{2} \\
-B & -A^{2}+D \nabla^{2}
\end{array}\right)
$$

Perturbations $\mathbf{u}$ are expanded in normal modes $\mathbf{u}$ $=\Sigma_{|\mathbf{k}|=k_{c}} \mathbf{u}_{0} \exp [\sigma(k) t+i \mathbf{k} \cdot \mathbf{r}]$ leading to the eigenvalue equation $\sigma(k)^{2}-\tau \sigma(k)+\Delta=0$ in which $\tau$ and $\Delta$ indicate the trace and the determinant of the linear operator $\mathcal{L}$, respectively. Depending on the values of the parameters $A$ and $D$ the system will undergo a Turing (stationary) bifurcation or a Hopf instability. As a matter of fact, 2D reaction-diffusion systems that display Turing patterns also present a Hopf bifurcation. The latter gives rise to oscillations if $\eta \equiv \sqrt{1 / D}$ $>\left(\sqrt{1+A^{2}}-1\right) / A[14]$. Otherwise, a Turing instability takes place, the case we focus on hereafter.

The marginal condition $(\sigma=0)$ leads to a curve $B$ $=B(k)$ the minimum of which yields the critical point $\left(B_{c}, k_{c}\right)=\left[(1+A \eta)^{2}, \sqrt{A \eta}\right]$. To describe naturally the separation from the critical values the rescaled parameter $\mu$ $=\left(B-B_{c}\right) / B_{c}$ dubbed supercriticality, replaces $B$ as the control parameter.

Before going to a perturbative analysis let us show examples of Turing patterns from direct simulations of the model in Fig. 1, similar to that obtained by other authors $[8,9]$. They correspond to hexagons with different phases and stripes, for different values of the supercriticality $\mu$. These patterns are shown here just as a guide to the ensuing weakly nonlinear analysis. (Technical details on the simulations will be given in Sec. V C.)

\section{WEAKLY NONLINEAR THEORY}

Amplitude equations are a classical tool to describe excited states beyond linear analysis [15]. We sketch briefly the main steps in obtaining them. Just above threshold the eigenvalues of the critical modes are close to zero, so that they are slowly varying modes, whereas the off-critical modes relax quickly, so only perturbations with $k$ around $k_{c}$ have to be considered. The solution of Eq. (2) can be expanded as

$$
\mathbf{u}=\sum_{j=1}^{N} \mathbf{u}_{0}\left(A_{j} e^{i \mathbf{k}_{j} \cdot \mathbf{r}}+\text { c.c. }\right)
$$

where $\mathbf{u}_{0}=(1,-\eta(1+A \eta) / A)^{T}$ stands for the eigenvector of the linear operator. Here we use the standard multiple-scale analysis [15] in which the control parameter and the derivatives are expanded in terms of a small parameter $\epsilon$ and or- thogonality conditions (Fredholm alternative) are used at each order. In the neighborhood of the bifurcation point, the critical amplitudes $A_{j}$ follow the so-called normal forms. Their general form can be derived from standard techniques of symmetry-breaking bifurcations [16]. A normal form describes perfect extended patterns, but slight variations in the pattern can be included by means of spatial terms with the suitable symmetries, so one arrives at the so-called amplitude equations. We discuss in the following the form of these equations for different planforms.

Stripes are characterized by a single amplitude $A$ that evolves according to

$$
\tau_{0} \partial_{t} A=\mu A-g|A|^{2} A+\xi_{0}^{2}\left(\partial_{x}+\frac{1}{2 i k_{c}} \partial_{y}^{2}\right)^{2} A,
$$

which is the normal form for a supercritical bifurcation plus a term that accounts for spatial variations, known as the Newell-Whitehead-Segel equation [17] in convection phenomena.

Squares are formed by two perpendicular sets of rolls with equal wave numbers, i.e., (a) $\mathbf{k}_{1} \perp \mathbf{k}_{2}$ and (b) $\left|k_{1}\right|=\left|k_{2}\right|$. They display four peaks with $\mathcal{D}_{4}$ symmetry in Fourier space. When condition (b) does not hold one obtains rectangular or bimodal patterns; instead, without the condition (a) rhombs (rhombic cells) arise. Squares or rhombs should obey coupled equations of the kind: $\tau_{0} \partial_{t} A_{1}=\mu A_{1}-g\left|A_{1}\right|^{2} A_{1}$ $-g_{\theta}\left|A_{2}\right| A_{1}$, in which $g_{\theta}$ depends on $\mathbf{k}_{1} \cdot \mathbf{k}_{2}$ (valid for angles not around $2 \pi / 3$ ).

Hexagons are built up by three modes satisfying the resonant condition $\mathbf{k}_{1}+\mathbf{k}_{2}+\mathbf{k}_{3}=\mathbf{0}$ (resonant triad), with $\left|k_{1}\right|$ $=\left|k_{2}\right|=\left|k_{3}\right|$. When the modes break the rotational symmetry but not reflexions $\left(\left|k_{1}\right| \neq\left|k_{2}\right|=\left|k_{3}\right|\right)$ squeezed hexagons result. Distortions breaking rotation and reflexion symmetries, $\left|k_{1}\right| \neq\left|k_{2}\right| \neq\left|k_{3}\right|$, lead to a pattern of sheared hexagons. Let us stress that what have been called "rhombs" or "rhombic cells" in many recent papers are actually distorted hexagons, a distinction far from semantic, because the term "rhomb" used for a three-mode pattern has induced some misleading interpretations [18]. Squares or true rhombs have never been observed in experiments, while slightly distorted hexagons have been reported in chemical systems [6] and in theoretical models $[11,19]$.

During the last years several authors have established that, up to the third order in the amplitudes, the general form of the amplitude equations for a hexagonal pattern should be as follows $[11,12]$ :

$$
\begin{aligned}
\tau_{0} \partial_{t} A_{1}= & \mu A_{1}+\xi_{0}^{2} \partial_{x_{1}}^{2} A_{1}+v \bar{A}_{2} \bar{A}_{3}-g\left|A_{1}\right|^{2} A_{1} \\
& -h\left(\left|A_{2}\right|^{2}+\left|A_{3}\right|^{2}\right) A_{1}+i \alpha_{1}\left[\bar{A}_{2} \partial_{x_{3}} \bar{A}_{3}+\bar{A}_{3} \partial_{x_{2}} \bar{A}_{2}\right] \\
& +i \alpha_{2}\left[\bar{A}_{2} \partial_{\tau_{3}} \bar{A}_{3}-\bar{A}_{3} \partial_{\tau_{2}} \bar{A}_{2}\right]
\end{aligned}
$$

where subindices in the derivatives stand for $\partial_{x_{i}}=\hat{\mathbf{n}}_{i} \cdot \nabla$ and $\partial_{\tau_{i}}=\hat{\boldsymbol{\tau}}_{i} \cdot \boldsymbol{\nabla}$, respectively, being $\hat{\mathbf{n}}_{i}$ the unitary vectors in the direction of $\mathbf{k}_{i}$ and $\hat{\boldsymbol{\tau}}_{i}$ orthogonal to $\hat{\mathbf{n}}_{i}$. Companion equations for $A_{2}$ and $A_{3}$ are simply obtained by subindex permutations. 
Although small, the quadratic term $\bar{A}_{2} \bar{A}_{3}$ ought to be included to ensure the bifurcation being subcritical. Consequently, terms in the form $\bar{A} \nabla \bar{A}$ [20] must also be considered. Notice that these terms are nonpotential, i.e., they cannot fit into a Lyapunov functional. In the following, we will assume $h, g>0$, which implies $A \eta<2.418$.

Exact expressions of coefficients in Eq. (6) are specific to the model. They have been calculated in the context of thermal fluid convection $[12,21,22]$ and flames fronts [23]. The linear coefficients stem simply from a Taylor expansion near onset of the growth parameter $\sigma(\mu, k)=\left(1 / \tau_{0}\right)\left[\mu-\xi_{0}^{2}(k\right.$ $\left.\left.-k_{c}\right)^{2}\right]$, that leads in our case to

$$
\tau_{0}^{-1}=\frac{1+A \eta}{1-\eta^{2}}, \quad \xi_{0}^{2}=\frac{4}{(1+A \eta)^{2}} .
$$

(These coefficients can be set to one after a suitable rescaling of time and space.) For the Brusselator nonlinear coefficients $v, g$, and $h$ were computed in Ref. [14]. We also gather here the expression for $\alpha_{1}$ and $\alpha_{2}$ [24],

$$
\begin{aligned}
v & =2 \frac{1-A \eta}{A(1+A \eta)}+\frac{2}{A} \mu \equiv v_{0}+v_{1} \mu, \\
g & =\frac{-8+38 A \eta+5 A^{2} \eta^{2}-8 A^{3} \eta^{3}}{9 A^{3} \eta(1+A \eta)}, \\
h & =\frac{-3+5 A \eta+7 A^{2} \eta^{2}-3 A^{3} \eta^{3}}{A^{3} \eta(1+A \eta)}, \\
\alpha_{1} & =\frac{4 \sqrt{A \eta}}{A(1+A \eta)^{2}}-\frac{1}{\sqrt{3}} \alpha_{2}, \\
\alpha_{2} & =\frac{-2 \sqrt{3} \sqrt{A \eta} \eta(1-A \eta)}{A^{2}\left[A^{2}+\eta^{2}(1+A \eta)^{2}\right]} .
\end{aligned}
$$

As distinguished from other systems, $v$ depends on $\mu$, so that we split $v$ in the form $v=v_{0}+v_{1} \mu$ [9]. Therefore, $v$ can change its sign depending on the values of $A \eta$. For $v>0$ the stationary solution consists of hexagons with a vanishing total phase ( $H_{0}$ hexagons), while for $v<0$ the total phase reaches $\pi$ ( $H_{\pi}$ hexagons).

The new terms with $\alpha_{1}$ and $\alpha_{2}$ deserve some comments. First, they correspond physically to wave number dilatations (term with $\alpha_{1}$ ) and stretch distortions (term with $\alpha_{2}$ ) in the pattern [22]. Second, the coefficient $\alpha_{2}$ remains rather small even for high values of $\eta$, while $\alpha_{1}$ is always positive and takes values of the same order as $v_{0}$, except for very small values of $\eta$.

In extended system, patterns issued from a random initial condition are often composed by several cellular domains with different orientations, which evolve through rearrangements of defects and domain walls. In general, such an evolution cannot be studied analytically, but its main ingredients would be made evident under simple situations. In the fol- lowing sections we will consider two such situations acting upon an almost perfect pattern: amplitude instabilities and phase instabilities.

\section{AMPLITUDE INSTABILITIES}

In this section we first analyze the stability of different symmetries as the control parameter is increased in Eq. (6). Although pure amplitude perturbations are rarely the most dangereous we complete the bifurcation analyses of previous papers $[8,9,14]$ by including off-critical wave numbers. We perform separately, computations for regular stripes and hexagons, and later on for mixed modes and distorted hexagons.

\section{A. Stripe and hexagon patterns}

Let us begin with the case of stripes with a wave number slightly off-critical $k=k_{c}+q$. These are solutions of Eq. (6) provided $A_{1}=\mathcal{S} e^{i q x_{1}}, A_{2}=A_{3}=0$, with $\mathcal{S}=\sqrt{\left(\mu-\xi_{0}^{2} q^{2}\right) / g}$. Considering uniform perturbations in the form $A_{1}=\mathcal{S}(1$ $\left.+r_{1}\right) e^{i q x_{1}}, A_{2}=r_{2} e^{i q x_{2}}$ and $A_{3}=r_{3} e^{i q x_{3}}$, the linear stability of this solution requires

$$
\lambda_{ \pm}=(g-h) \mathcal{S}^{2} \pm\left|v^{\prime}\right| \mathcal{S}<0,
$$

where $v^{\prime}=v+2 \alpha_{1} q$. The growth rate $\lambda_{ \pm}$vanishes along the curves

$$
\begin{aligned}
& \mu_{S \pm} \\
& =\frac{-\left(2 v_{0}^{\prime} v_{1}-g^{\prime}\right) \pm \sqrt{\left(2 v_{0}^{\prime} v_{1}-g^{\prime}\right)^{2}-4 v_{1}^{2}\left(v_{0}^{\prime}+g^{\prime} \xi_{0}^{2} q^{2}\right)}}{2 v_{1}^{2}},
\end{aligned}
$$

where $g^{\prime}=(g-h)^{2} / g$ and $v_{0}^{\prime}=v_{0}+2 \alpha_{1} q$. Thus, stripes are stable within the range $\mu_{S-}<\mu<\mu_{S+}$, i.e., in a closed area of the parameter space $(q, \mu)$. (Notice that $\alpha_{2}$ does not enter into this expression.)

Likewise, by setting $A_{i}=\mathcal{H} e^{i q x_{i}}$ the following eigenvalues are achieved in the hexagonal case:

$$
\begin{aligned}
\sigma_{1} & =v^{\prime} \mathcal{H}-2(g+2 h) \mathcal{H}^{2}, \\
\sigma_{2} & =\sigma_{3}=-2 v^{\prime} \mathcal{H}-2(g-h) \mathcal{H}^{2}, \\
\sigma_{p 1} & =-3 v^{\prime} \mathcal{H}, \\
\sigma_{p 2} & =\sigma_{p 3}=0,
\end{aligned}
$$

where $\mathcal{H}$ is the uniform and stationary solution of Eq. (6) given by

$$
\mathcal{H}=\frac{v^{\prime} \pm \sqrt{v^{\prime 2}+4(g+2 h)\left(\mu-\xi_{0}^{2} q^{2}\right)}}{2(g+2 h)} .
$$

Equation (13) shows that the total phase is a damped mode that relaxes monotonically towards $0\left(H_{0}\right)$ or $\pi\left(H_{\pi}\right)$ depending on the sign of $v^{\prime}$. Thus, initially disordered patterns will evolve until the maxima (minima) of $X$ arrange themselves on a honeycomb lattice of $H_{0}$ hexagons $\left(H_{\pi}\right.$ hexagons) for $v^{\prime}>0\left(v^{\prime}<0\right)$. We can deal with both solutions simply by using $\left|v^{\prime}\right|$. Examples of these two kinds of hexagons can be seen in simulations in Figs. 1(a) and 1(c), 
respectively. Let us mention that $H_{0}$ hexagons are similar to up hexagons (hexagons with upflow currents in the center), while $H_{0}$ hexagons are analogous to down hexagons in convection.

The first two eigenvalues in Eqs. (11), (12) correspond to the amplitude modes. For hexagons to be stable, they must be negative, i.e.,

$$
\begin{gathered}
w=2(g+2 h) \mathcal{H}^{2}-\left|v^{\prime}\right| \mathcal{H}>0, \\
u=(g-h) \mathcal{H}^{2}+\left|v^{\prime}\right| \mathcal{H}>0 .
\end{gathered}
$$

The first inequality represents the marginal condition, i.e., hexagons appear whether $\mu>\mu_{M}$,

$$
\mu_{M}=\frac{-2\left[v_{0}^{\prime} v_{1}+2(g+2 h)\right]^{2} \pm \sqrt{4\left[v_{0}^{\prime} v_{1}+2(g+2 h)\right]^{2}-4 v_{1}^{2}\left[v_{0}^{\prime 2}-4(g+2 h)^{2}\right]}}{2 v_{1}^{2}} .
$$

The function $u$ has two real roots corresponding to the following curves:

$$
\mu_{H \pm}=\frac{-\left(2 v_{0}^{\prime} v_{1}-\frac{(g-h)^{2}}{(2 g+h)}\right) \pm \sqrt{\left(2 v_{0}^{\prime} v_{1}-\frac{(g-h)^{2}}{(2 g+h)}\right)^{2}-4 v_{1}^{2}\left(v_{0}^{\prime 2}+\frac{(g-h)^{2}}{2 g+h} \xi_{0}^{2} q^{2}\right)}}{2 v_{1}^{2}}
$$

and hexagons become unstable within $\mu_{H_{-}}<\mu<\mu_{H+}$. The fact that there are two branches for the instability limits, $\mu_{S \pm}$ and $\mu_{H \pm}$, is a result of quadratic coefficient depending on the control parameter for this particular model.

We discuss first the standard case $q=0$. Stability regions are shown as functions of $(A \eta, \mu)$ in Fig. 2. Dashed regions correspond to stable stripes and shaded areas to hexagonal stable solutions $\left(H_{0}\right.$ and $H_{\pi}$ in bright and dark gray, respectively). The curves for the amplitude instabilities are represented with solid $\left(\mu_{S \pm}\right)$ and dashed lines $\left(\mu_{M}, \mu_{H \pm}\right)$. Stripes can be stable only if $A \eta>0.879$ (line 1), while hexagons are always stable when $A \eta<0.958$ (line 2). Line 3 is the limit for the amplitude equations to be valid $(g>0)$. Three different regions can be distinguished when $\mu$ is increased. In region I $(0<A \eta<0.879)$, the condition (17) is satisfied and consequently $H_{0}$ hexagons are always stable above onset. In

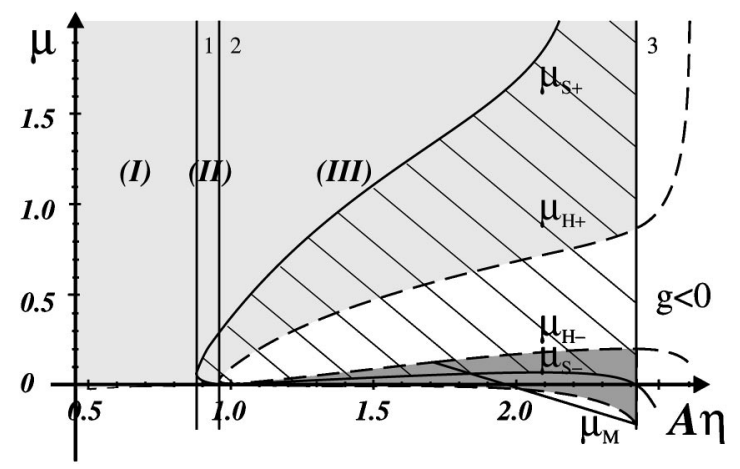

FIG. 2. Stability thresholds in the parameter space $(A \eta, \mu)$ for Turing patterns. Shaded and striped regions denote stable hexagons and bands, respectively. In region I only hexagons are stable. Hexagons and bands coexist for $\mu_{S-}<\mu<\mu_{H_{-}}$in region II. In region III hexagons are unstable within the range $\mu_{H+}<\mu<\mu_{S+}$. To the right of line $3, g<0$ and, therefore, terms higher than cubic are needed in the amplitude equation. region II $(0.879<A \eta<0.958)$, the hexagonal solution remains stable; however, on increasing $\mu$ stripes also become stable, and the two planforms coexist within $\mu_{S-}<\mu$ $<\mu_{S+}$. In region III $(0.953<A \eta<2.418)$, an interesting phenomenon occurs: the hexagonal solution reappears after stripes [14]. These are called reentrant hexagons, which have also been observed in experiments [25]. Within the range $0.9583<A \eta<1$ reentrant hexagons have the same phase as critical ones ( $H_{0}$ hexagons), whereas for $A \eta>1 H_{\pi}$ hexagons grow near threshold, while the reentrant ones change their phase to $H_{0}$ hexagons. (Bifurcation diagrams for these situations were reported in Ref. [9]).

When spatial disturbaces are included $(q \neq 0)$ the amplitude stability curves are represented in terms of $\mu$ and $q$. In Fig. 3 stability diagrams for characteristic values of $A \eta$ inside the three main regions in Fig. 2 are shown. Let us mention that the stability regions bent to the left and the thresholds are significantly modified with regard to the case with $\alpha_{i}=0$ [24]. As we will show, this asymmetry is crucial in order to fit with numerical simulations.

\section{B. Mixed modes and distorted hexagons}

In some experiments a mixture of hexagons and rolls, dubbed mixed modes has been reported [26]. So, naturally we must analyze the stability of a solution

$$
A_{1}=\frac{g|v|}{h-g}, \quad A_{2}=A_{3}=\left(\frac{\mu-g A_{1}^{2}}{g+h}\right)^{1 / 2}
$$

which exists beyond the stability limit for stripes $\left(\mu>\mu_{s}\right)$. These modes are always unstable under amplitude perturbations, but the mixed modes observed in CIMA reaction could have arisen from 3D effects, namely, by superposition of two 


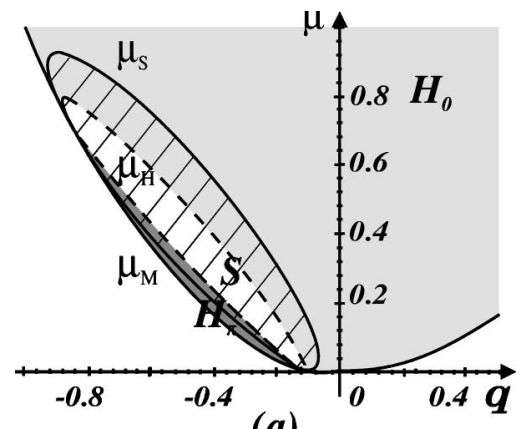

(a)

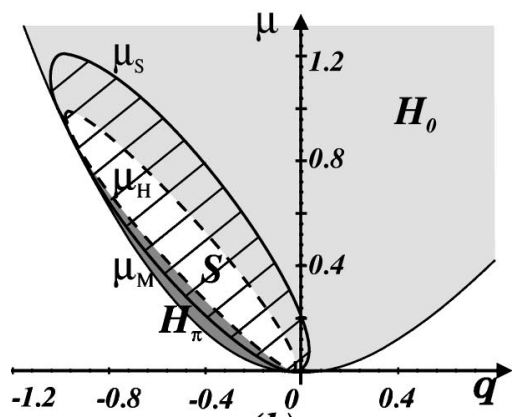

(b)

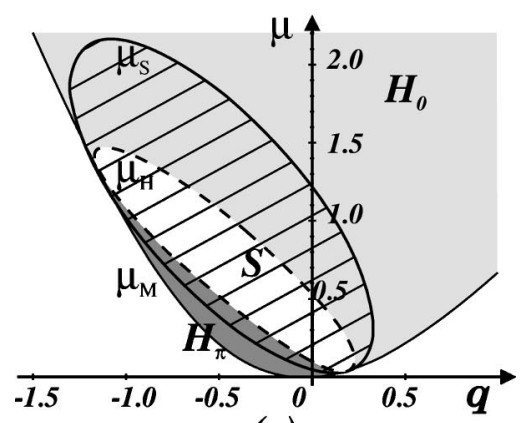

(c)

FIG. 3. Stability diagrams taking into account spatial quadratic terms. Parameter values: (a) $A \eta=0.8, \quad \alpha_{1}=0.22, \quad \alpha_{2}=-2.4$ $\times 10^{-4}$; (b) $A \eta=0.92, \quad \alpha_{1}=0.22, \quad \alpha_{2}=-1.3 \times 10^{-4}$; (c) $A \eta$ $=1.59, \alpha_{1}=0.21, \alpha_{2}=-2.7 \times 10^{-3}$.

Turing layers, a situation not covered by our description. Moreover, neither squares nor rhombs are stable for the Brusselator [9] or for any two-variable reaction-diffusion system [18].

Other possible solutions are distorted hexagons. In the following computations we consider only the simple case of squeezed hexagons for which analytical calculations can be made. (An interested reader can find useful results on the bifurcation analysis for sheared hexagons in Refs. [27,19]). Let us consider tilted solutions given by the transformation $(x, y) \rightarrow[x,(1+\Delta) y]$, which reduces the symmetry group from $\mathcal{D}_{6}$ to $\mathcal{D}_{2}$. Then, the stationary solution of Eq. (6) can be written in terms of the amplitudes $A_{1}=\mathcal{A}, A_{2}=\mathcal{B} e^{i \delta}, A_{3}$ $=\mathcal{B} e^{-i \delta}$ provided

$$
\begin{gathered}
\mu \mathcal{A}+\left[v+\delta\left(\sqrt{3} \alpha_{1}-\alpha_{2}\right)\right] \mathcal{B}^{2}-\left(g \mathcal{A}+2 h \mathcal{B}^{2}\right) \mathcal{A}=0, \\
\mu-\frac{3}{4} \delta^{2}+\left[v+\frac{1}{2} \delta\left(\sqrt{3} \alpha_{1}+\alpha_{2}\right)\right] \mathcal{A}-(g+h) \mathcal{B}^{2}-h \mathcal{A}^{2}=0 .
\end{gathered}
$$

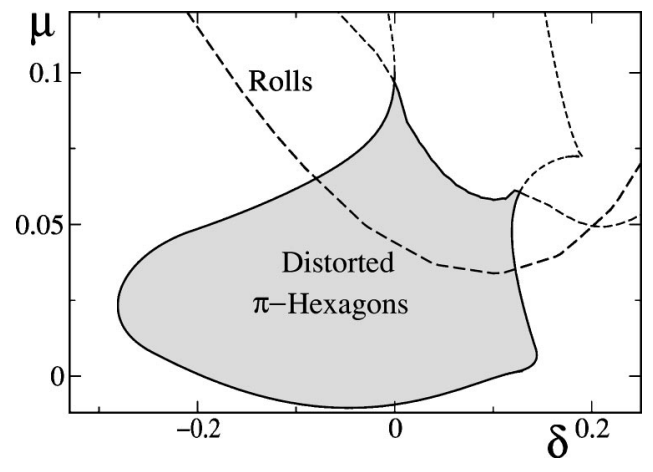

FIG. 4. Amplitude stability zone for squeezed hexagons (shaded region). Above the lower dashed line, stripes are also stable. Parameter values: $A \eta=1.59, \alpha_{1}=0.21, \alpha_{2}=-2.7 \times 10^{-3}$.

The latter expression gives $\mathcal{B}^{2}=\left\{\mu-\frac{3}{4} \delta^{2}+\left[v+\frac{1}{2} \delta\left(\sqrt{3} \alpha_{1}\right.\right.\right.$ $\left.\left.\left.+\alpha_{2}\right)\right] \mathcal{A}-h \mathcal{A}^{2}\right\} /(g+h)$, which introduced into the former relationship leads to a cubic equation [27]

$$
a_{3} \mathcal{A}^{3}+a_{2} \mathcal{A}^{2}+a_{1} \mathcal{A}+a_{0}=0
$$

with the coefficients

$$
\begin{aligned}
a_{3}= & 2 h^{2}-g(g+h), \\
a_{2}= & -h\left[3 v+2 \delta \sqrt{3} \alpha_{1}\right], \\
a_{1}= & -\mu(h-g)-\frac{3}{2} h \delta^{2} \\
& +\left[v+\delta\left(\sqrt{3} \alpha_{1}-\alpha_{2}\right)\right]\left[v+\frac{1}{2} \delta\left(\sqrt{3} \alpha_{1}+\alpha_{2}\right)\right], \\
a_{0}= & \left(\mu-\frac{3}{4} \delta^{2}\right)\left[v+\delta\left(\sqrt{3} \alpha_{1}-\alpha_{2}\right)\right] .
\end{aligned}
$$

Stability curves for squeezed hexagons are shown in the $(\mu, \delta)$ plane for $A \eta=1.59$ in Fig. 4 . We only show $H_{\pi}$ hexagons, but as for regular hexagons [Fig. 3(c)], the stability region of squeezed $H_{0}$ hexagons stands for higher values of $\mu$. Squeezed hexagons are stable within the shaded area and coexist with stripes above the dashed curve. The minima of the stability curves for stripes and hexagons (dashed lines) are shifted to the left, owing to the sign of $\alpha_{2}$. Moreover, distorted hexagons are not fully replaced by stripes just over one point but on a wide interval in this case. (Details are given elsewhere [28].)

\section{LONG-WAVELENGTH INSTABILITIES}

A more interesting kind of instabilities occurs because of spatial modulations of the underlying modes. Since a perfect pattern remains invariant under spatial translations along some symmetry axes, slight long-wavelength variations will evolve in a slow scale, according to the so-called phase equation. This formalism applies unless the gradient of phase becomes large at some location, i.e, at the core of a defect where the phase is singular. For the sake of clarity, we discuss separately this equation for patterns of stripes and hexagons, and for distorted hexagons. 


\section{A. Stripe and hexagonal patterns}

Let us briefly recall the phase equation for stripes. It is obtained by considering small amplitude $(r)$ and phase $(\phi)$ perturbations on the solution $\mathcal{S}, A=\mathcal{S}(1+r+i \phi) e^{i q x}$. This leads to coupled equations for $r$ and $\phi$. Consistently with the phase formalism, $r$ is eliminated adiabatically and written in terms of $\phi$, so that we end up with the phase equation $\partial_{t} \phi$ $=D_{\|} \partial_{x}^{2} \phi+D_{\perp} \partial_{y}^{2} \phi$, with $D_{\|}=\left(\mu-3 \xi_{0}^{2} q^{2}\right) /\left(\mu-\xi_{0}^{2} q^{2}\right), D_{\perp}$ $=\xi_{0} q / k_{c}[15]$. The zig-zag instability becomes dangereous whenever $D_{\perp}<0$, i.e., for $k>k_{c}$, whereas the Eckhaus instability takes place when $D_{\|}<0$, i.e, for $k-k_{c}>\sqrt{\mu / 3 \xi_{0}^{2}}$.

Generically hexagons have two independent marginal directions and, therefore, long wavelength perturbations result in a 2D phase equation. The form of this equation can be deduced solely by symmetry arguments [29] (see next section). To compute the coefficients we consider perturbations in Eq. (15) in the form

$$
A_{i}=\mathcal{H}\left(1+r_{i}+i \phi_{i}\right) e^{i q x_{i}}, \quad i=1,2,3,
$$

so that they evolve according to

$$
\begin{gathered}
\partial_{T} r_{1}=\left(\mu-q^{2}\right) r_{1}+\partial_{x_{1}}^{2} r_{1}-2 q \partial_{x_{1}} \phi_{1}+\left|v+2 q \alpha_{1}\right| \mathcal{H}\left(r_{2}+r_{3}\right) \\
-2 h \mathcal{H}^{2}\left(r_{1}+r_{2}+r_{3}\right)-3 g \mathcal{H}^{2} r_{1} \\
+\alpha_{1} \mathcal{H}\left(\partial_{x_{2}} \phi_{2}+\partial_{x_{3}} \phi_{3}\right)+\alpha_{2} \mathcal{H}\left(\partial_{\tau_{3}} \phi_{3}-\partial_{\tau_{2}} \phi_{2}\right), \\
\partial_{T} \phi_{1}=\left(\mu-q^{2}\right) \phi_{1}+\partial_{x_{1}}^{2} \phi_{1}+2 q \partial_{x_{1}} r_{1} \\
\quad-\left|v+2 q \alpha_{1}\right| \mathcal{H}\left(\phi_{2}+\phi_{3}\right)-(g+2 h) \mathcal{H}^{2} \phi_{1} \\
+\alpha_{1} \mathcal{H}\left(\partial_{x_{2}} r_{2}+\partial_{x_{3}} r_{3}\right)+\alpha_{2} \mathcal{H}\left(\partial_{\tau_{3}} r_{3}-\partial_{\tau_{2}} r_{2}\right) .
\end{gathered}
$$

These expressions can be further reduced looking back to the eigenvalues in Eqs. (11)-(14). The three $\sigma_{i}$ are associated to the eigenvectors $\vec{v}_{1}=(1,1,1), \vec{v}_{2}=(1,-1 / 2,-1 / 2)$ and $\vec{v}_{3}=(0, \sqrt{3} / 2,-\sqrt{3} / 2)$ and determine the amplitude stability. The fourth one (labeled $\sigma_{p 1}$ ) (always negative) corresponds to the total phase $\Phi=\phi_{1}+\phi_{2}+\phi_{3}$ decaying to 0 or $\pi$. So the phase evolution results from the other two eigenvalues after using the following arguments. The amplitudes remain invariant under shifts $\Delta x=2 n \lambda$ or $\Delta y=2 n \lambda / \sqrt{3}$, i.e, under a phase change $\left(\phi_{1}^{\prime}, \phi_{2}^{\prime}, \phi_{3}^{\prime}\right)=\left(\phi_{1}, \phi_{2}, \phi_{3}\right)+k \Delta x \mathbf{v}_{2}$ $+k \Delta y \mathbf{v}_{3}$, so that the eigenvectors $\mathbf{v}_{2}$ and $\mathbf{v}_{3}$ are marginal modes (Goldstone modes). In view that $\Phi$ can be kept constant only two phases are independent, so it is useful to consider $\phi_{x}=-\left(\phi_{2}+\phi_{3}\right)$ and $\phi_{y}=\left(\phi_{2}-\phi_{3}\right) / \sqrt{3}$. Adiabatical elimination of enslaved modes and a center manifold reduction to $\left(\phi_{x}, \phi_{y}\right)$ gives $[30,31,22,24]$

$$
\partial_{T} \boldsymbol{\phi}=D_{t} \nabla^{2} \boldsymbol{\phi}+\left(D_{l}-D_{t}\right) \boldsymbol{\nabla}(\boldsymbol{\nabla} \cdot \boldsymbol{\phi}),
$$

with the coefficients [22]:

$$
D_{t}=\frac{1}{4}-\frac{\xi_{0}^{2} q^{2}}{2 u}+\frac{\mathcal{H}^{2}}{8 u}\left(\alpha_{1}-\sqrt{3} \alpha_{2}\right)^{2},
$$

$$
\begin{aligned}
D_{l}= & \frac{3}{4}-\frac{\xi_{0}^{2} q^{2}(4 u+w)}{2 u w}+\frac{\mathcal{H}^{2}}{8 u}\left(\alpha_{1}-\sqrt{3} \alpha_{2}\right)^{2} \\
& -\frac{\alpha_{1} \mathcal{H}^{2}}{w}\left(\alpha_{1}+\sqrt{3} \alpha_{2}\right)+\frac{\xi_{0} q \mathcal{H}}{w}\left(3 \alpha_{1}+\sqrt{3} \alpha_{2}\right),
\end{aligned}
$$

[ $u$ and $w$ were given in Eqs. (16), (17)]. This phase equation is similar to the wave equation of an isotropic solid [30]. Hence the phase field can be split into two normal modes $\boldsymbol{\phi}=\boldsymbol{\phi}_{l}+\boldsymbol{\phi}_{t}$, that obey the relationships $\boldsymbol{\nabla} \times \boldsymbol{\phi}_{l}=\mathbf{0}$ and $\boldsymbol{\nabla}$ - $\boldsymbol{\phi}_{t}=\mathbf{0}$, respectively. Squeezed $\phi_{l}$ and sheared $\phi_{t}$ distortions relax diffusively $\left(\partial_{t} \boldsymbol{\phi}_{l, t}=D_{l, t} \nabla^{2} \boldsymbol{\phi}_{l, t}\right)$ inside the zones bounded by $D_{l, t}=0$. We will comment on the phase instability regions in connection with numerical computations in Sec. V C.

\section{B. Squeezed hexagonal patterns}

After applying the same procedure to solutions of Eq. (22) and restricting the analysis to the linear part at lowest order one obtains an equation of the form [29]

$$
\dot{\phi}_{i}=\mathcal{D}_{i}^{k l j} \partial_{k l}^{2} \phi_{j}
$$

The reflection symmetries $x \rightarrow-x$ and $y \rightarrow-y$ and $k \leftrightarrow l$ leave six independent coefficients that can be written in a matricial form $\partial_{t} \boldsymbol{\phi}=\mathcal{B} \cdot \boldsymbol{\phi}$, with

$$
\mathcal{B} \equiv\left(\begin{array}{cc}
D_{1} \partial_{x}^{2}+D_{2} \partial_{y}^{2} & D_{5} \partial_{x y}^{2} \\
D_{6} \partial_{x y}^{2} & D_{3} \partial_{x}^{2}+D_{4} \partial_{y}^{2}
\end{array}\right)
$$

This is the general expression for the phase of distorted hexagons or for a rhombic pattern. For squares the diagonal symmetry $x \rightarrow y$ gives in addition $D_{1}=D_{4}, D_{2}=D_{3}, D_{5}=D_{6}$, so that only three diffusion coefficients should remain independent. Finally, rotation symmetry reduces these to two independent coefficients for equilateral hexagons, because $D_{1}$ $=D_{4}=D_{l}, D_{3}=D_{5}=D_{t}$ and $D_{5}=D_{6}=D_{l}-D_{t}$, so that Eq. (25) is recovered. In the case $\alpha_{i}=0$ the diffusion coefficients $D_{i}$ for squeezed hexagons can be computed from Eq. (6). Their explicit expressions are rather cumbersome, but an interested reader can find them in Ref. [28]. The eigenvalues of $\mathcal{B}$ has to be computed numerically and they give the stability curves in Fig. 5, now drawn in terms of the deviations $\Delta \theta$ from $2 \pi / 3 \mathrm{rad}$. This stability region (dashed region) is similar to, though smaller than the region determined numerically in Ref. [11]. [Its width is model dependent (see Ref. [28]).]

When $\alpha_{1}=\alpha_{2}=0$, Eqs. (6) become variational, i.e., a Lyapunov functional can be derived. Equilateral hexagons get the minimum energy among the squeezed lattices and, therefore, this should be the selected planform. Nevertheless, though globally unstable, distorted hexagons can be locally stable owing to boundary conditions or pinning (nonadiabatic) effects among domains (a situation not covered within the amplitude equation framework), as obtained in experiments. Irregular patterns come out spontaneously from the homogeneous state after making a sudden jump in control 


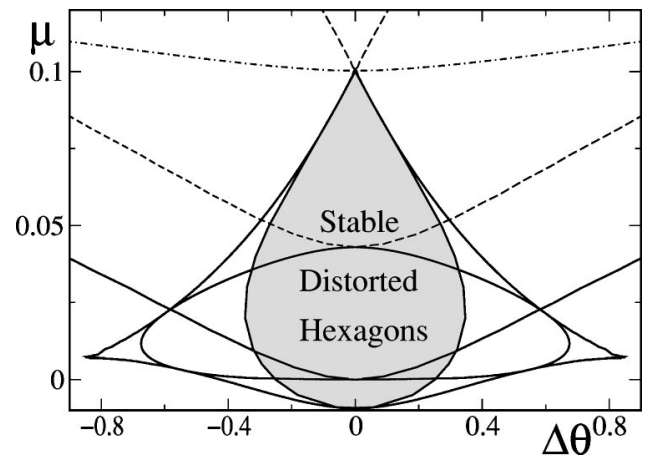

FIG. 5. Stability regions of hexagons distorted an angle $\Delta \theta$ as a function of $\mu$.

parameter, so that the Turing stability region is reached, or by illuminating the cell through a distorted hexagonal lattice of spots with angles slightly different from $2 \pi / 3$. In both cases the evolved pattern displays several domains with unequal angles. The rms $\Delta \theta_{r m s}$ of the observed angular distribution function changes with $\mu$ in qualitative accord with the theoretical findings $[11,26]$.

\section{Comparison with numerical simulations}

The validity of these stability diagrams have been checked against simulations of model (1). These are performed after discretizing the spatial terms in a square mesh of $128 \times 128$ points with periodic boundary conditions. Similar outputs are obtained using two different integration methods: a spectral method [32] and a semi-implicit method [33]. During the first stages several domains, separated by some walls or defects, are formed, but most of these evolve slowly. After about 5000 time steps a stationary pattern with few defects is eventually reached. Typical stationary snapshots arising from random initial conditions for several values of $\mu$ are shown in Fig. 6. Values for the concentration $x$ are represented in a gray scale varying from black (minimum) to white (maximum). On varying the control parameter $\mu$ the

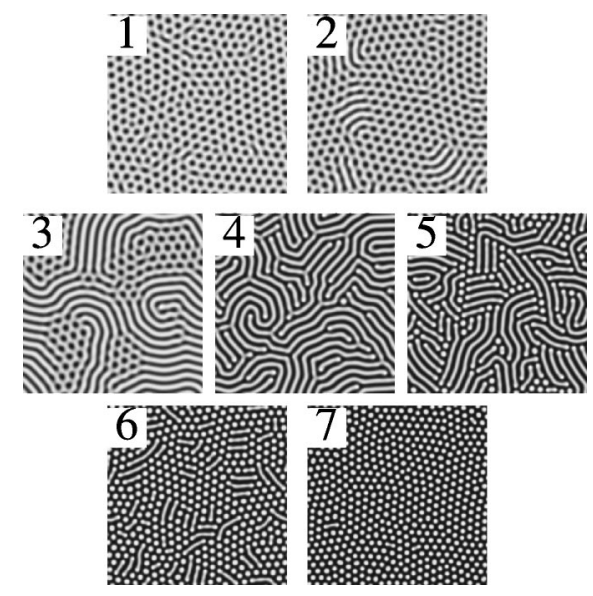

FIG. 6. Patterns from computations of the Brusselator. Parameter values $A \eta=1.59$ and (1) $\mu=0.04$, (2) $\mu=0.07$, (3) $\mu=0.08$, (4) $\mu=0.30$, (5) $\mu=0.54$, (6) $\mu=0.66$, and (7) $\mu=0.98$.

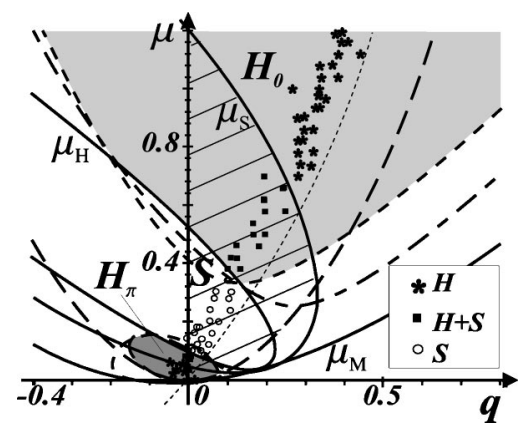

FIG. 7. Amplitude and phase instabilities (dashed curves) for $A \eta=1.59, \alpha_{1}=0.21, \alpha_{2}=-2.7 \times 10^{-3}$. -- Eckhaus instability, - - - curve $D_{l}=0$, and - - $D_{t}=0$. Dark areas: stable hexagons. Striped domains: stable stripes. Points: numerical simulations. Dotted line: maximum linear growth.

sequence $H_{\pi}$ hexagons (asterisk) $\rightarrow H_{\pi}$-hexagon-stripe mixtures (full squares) $\rightarrow$ stripes (open circles), $\rightarrow H_{0}$-hexagon-stripe mixtures (full squares) $\rightarrow H_{0}$ hexagons (asterisk) is observed. From the Fourier transform of the pattern one obtains the average wave number that increases with $\mu$, its value remaining not far from the line of highest linear growth (dotted line). These fit rather well into the stability regions of the asymmetrical diagram (Fig. 7), hence confirming that the spatial nonlinear terms are crucial for a correct interpretation of the transition between different symmetries and the nonlinear pattern selection [24].

Finally, we performed a simulation starting from a given initial pattern of stripes inside the region $S$ in Fig. 7. A sudden decreasing of $\mu$ to a value for which $H_{\pi}$ hexagons are stable, gives rise to a pattern of steady sheared hexagons, as one can see in the Fourier spectrum in Fig. 8. Although, the sheared case has not been worked out explicitly, this simulation confirms that initial and/or boundary conditions can give rise to locally stable distorted hexagons, as discussed in Sec. V B.

\section{CONCLUSIONS}

We have presented a weakly nonlinear analysis for the Turing patterns formed in the Brusselator model, for which we have derived amplitude equations for the excited modes. Up to third order in the amplitudes these equations also include spatial nonlinear terms, as it has also been discussed
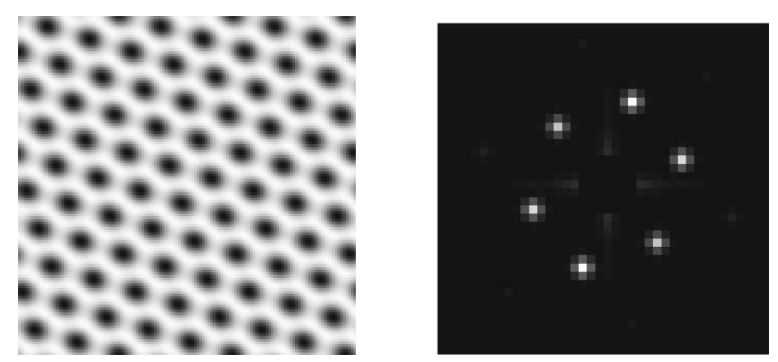

FIG. 8. A stable distorted hexagon pattern that comes up from simulation of the Brusselator and its ensuing Fourier spectrum. 
in other systems. The coefficients of these generalized amplitude equations have been explicitly calculated, allowing us to determine the relative stability of hexagons and stripes near the onset of the Turing instability. From those equations the evolution of phase modes has been derived [34]. The diffusion coefficients of the phase equations, together with the eigenvalues of amplitude equations allow us to calculate the stability regions for stripes and hexagons in parameter space. These regions look quite different whether the spatial nonlinear terms are included in the description.

We have computed the phase instability regions of stretched $H_{\pi}$ hexagons. Although globally unstable, stretched hexagons can be locally stable with distortion angles inside the phase stability region. This is qualitatively similar to the stability diagram reported in experiments on initially imposed distorted chemical patterns $[11,26]$, but the permitted angles for the Brusselator result to be rather small.

To confirm these analytical findings numerical simulations of the Brusselator model have also been performed. The average wave number of patterns in these simulations fits quite well into the stability regions calculated from the amplitude equation. This fact gives valuable support to include nonpotential terms into evolution equations. We also checked that simulations from suitable initial conditions can lead to stable distorted hexagons in the Brusselator, as found by other authors [20]. We guess the results presented here will stimulate a search of spatial effects in Turing patternforming experiments.

\section{ACKNOWLEDGMENTS}

We wish to thank B. Echebarria (Northeastern University), H. Mancini, M. L. Ramón (Universidad de Navarra, Pamplona), and A. Pérez-Muñuzuri (Universidad de Santiago de Compostela), for helpful comments and discussions. This work was partially supported by the DGICYT (Spanish Government) Grant No. PB98-0578, the PIUNA (Universidad de Navarra) and Integrated Action Italy-Spain HI9730. B.P. acknowledges the financial support from "Asociación de Amigos de la Universidad de Navarra" and "Caja de Ahorros de Pamplona.'
[1] A.M. Turing, Philos. Trans. R. Soc. London, Ser. B 327, 37 (1952).

[2] V. Castets, E. Dulos, J. Boissonade, and P. De Kepper, Phys. Rev. Lett. 64, 2953 (1990).

[3] I. Epstein, I. Lengyel, S. Kadar, M. Kagana, and M. Yokoyama, Physica A 188, 26 (1992).

[4] A.K. Horváth, M. Dolnik, A.P. Muñuzuri, A.M. Zhabotinsky, and I.R. Epstein, Phys. Rev. Lett. 83, 2950 (1999).

[5] M. Watzel and A.F. Münster, Chem. Phys. Lett. 242, 273 (1995); A.F. Münster, M. Watzel, and F.W. Schneider, Phys. Scr. T67, 58 (1996); H. Orban, K. Kurin-Csörgei, A.M. Zhabotinsky, and I.R. Epstein, J. Phys. Chem. B 103, 36 (1999); O. Steinbock, E. Kasper, and S.C. Müller, J. Phys. Chem. A 103, 3442 (1999).

[6] Q. Ouyang and H.L. Swinney, Nature (London) 352, 610 (1991).

[7] I. Prigogine and R. Lefever, J. Chem. Phys. 48, 1695 (1968); G. Nicolis and I. Prigogine, Self-Organization in Nonequilibrium Systems (Wiley, New York, 1977).

[8] V. Dufiet and J. Boissonade, J. Chem. Phys. 96, 1 (1992); V. Dufiet, Ph. D. thesis, Université de Bordeaux I, 1994.

[9] A. De Wit, Thèse doctoral, Université Libre de Bruxelles, 1993; in Advances in Chemical Physics, edited by I. Prigogine and S.A Rice (Wiley, New York, 1999), Vol. 109, p. 435.

[10] E. Mosekilde, F. Larsen, G. Dewel, and P. Borckmans, Int. J. Bifurcation Chaos Appl. Sci. Eng. 8, 1003 (1998); K.E. Rasmussen, W. Mazin, E. Mosekilde, G. Dewel, and P. Borckmans, ibid. 6, 1077 (1996).

[11] Q. Ouyang, G. Gunaratne, and H. Swinney, Chaos 3, 707 (1993); G.H. Gunaratne, Q. Ouyang, and H.L. Swinney, Phys. Rev. E 50, 2802 (1994).

[12] H. Brand, Prog. Theor. Phys. Suppl. 99, 442 (1989); E.A. Kuznetsov, A.A. Nepomnyashchy, and L.M. Pismen, Phys. Lett. A 205, 261 (1995).
[13] F.H. Busse, Rep. Prog. Phys. 41, 1929 (1978).

[14] J. Verdasca, A. de Wit, G. Dewel, and P. Borckmans, Phys. Lett. A 168, 194 (1992).

[15] P. Manneville, Structures Dissipatives, Chaos et Turbulence (Aléa Saclay, Paris, 1991); D. Walgraef, Spatio-Temporal Pattern Formation (Springer, New York, 1997).

[16] H.F. Golubitsky, J.W. Swift, and E. Knobloch, Physica D 10, 249 (1984).

[17] A.C. Newell and J.A. Whitehead, J. Fluid Mech. 38, 279 (1969); L.A. Segel, ibid. 38, 203 (1969). .

[18] S.L. Judd and M. Silber, Physica D 136, 45 (2000).

[19] V. Pérez-Muñuzuri, M Gómez-Gesteira, A.P. Muñuzuri, L.O. Chua, and V. Pérez-Villar, Physica D 82, 195 (1995); R. Schmitz and W. Zimmermann, J. Phys. II 7, 677 (1997); P.C. Matthews, Physica D 116, 81 (1998).

[20] The problem has two small parameters $A$ and the subcriticality $v$. Our analysis includes terms up to $O\left(A^{3} v\right)$.

[21] J. Bragard, Ph. D. thesis, Universidad Compdutense de Madrid, 1996; A.A. Golovin, A.A. Nepomnyashchy, and L.M. Pismen, J. Fluid Mech. 356317 (1997).

[22] B. Echebarria and C. Pérez-García, Europhys. Lett. 43, 35 (1998).

[23] R. Kuske and P. Milewski, Eur. J. Appl. Math. 10, 157 (1999).

[24] B. Peña and C. Pérez-García, Europhys. Lett. 51, 300 (2000).

[25] E. Dulos, P. Davies, B. Rudovics, and P. De Kepper, Physica D 98, 53 (1996).

[26] Q. Ouyang and H.L. Swinney, in Chemical Waves and Patterns, edited by R. Kapral and K. Showalter (Kluwer, Dordrecht, 1995).

[27] B.A. Malomed, A.A. Nepomnyashchy, and A.E Nuz, Physica D 70, 357 (1994).

[28] B. Peña and C. Pérez-García, Int. J. Bifurcation Chaos Appl. Sci. Eng. (to be published).

[29] S. Zaleski, Thèse 3ème cycle, Université de Paris VI, 1980. 
[30] J. Lauzeral, S. Mètens, and D. Walgraef, Europhys. Lett. 24, 707 (1993).

[31] R. Hoyle, Appl. Math. Lett. 9, 81 (1995).

[32] M. Bestehorn (private communication).
[33] V. Dufiet and J. Boissonade, Physica A 188, 158 (1992).

[34] A full analysis of the phase dynamics, without passing through the amplitude equations, can lead to a bit different $D_{\perp}(q)$, as discussed in Ref. [15]. 